Revista de Matemática: Teoría y Aplicaciones 2004 11(2) : 1-23

CIMPA - UCR - CCSS ISSN: 1409-2433

\title{
INVARIANT MANIFOLDS IN PARAMETRIC TURBULENT MODELS *
}

\author{
Vladimir N. GrebeneV ${ }^{\dagger} \quad$ G.G. ChernyKH ${ }^{\ddagger}$
}

Received/Recibido: 20 May 2003

\begin{abstract}
The article is devoted to examining the so-called local-equilibrium approximations used while modeling turbulent flows. The dynamics of a far plane turbulent wake are investigated as an example. In this article, we analyze these approximations by using the method of differential constraints. We show that some algebraic models based on using the local-equilibrium approximation can be interpreted as equations of invariant manifolds generated by the models under consideration. Reduction of the models on the corresponding invariant manifolds made it possible to find self-similar solutions and to separate explicit solutions. Moreover, some empirical constants may be calculated and their obtained values are close to the recommended quantities.
\end{abstract}

Keywords: local-equilibrium approximation, turbulent wake, method of differential constrains, invariant manifold, self-similar solutions.

\section{Resumen}

El artículo trata de examinar el llamado equilibrio local de aproximaciones usado cuando se modelan fluidos turbulentos. La dinámica de una estela turbulenta plana es estudiada como un ejemplo. Analizamos estas aproximaciones usando el método de restricciones diferenciales. Mostramos que algunos modelos algebraicos basados en el uso de la aproximación de equilibrio local pueden ser interpretadas como ecuaciones de variedades invariantes generadas por los modelos bajo consideración. La reducción de los modelos a las variedades correspondientes hicieron posible encontrar soluciones auto-similares y separar soluciones explícitas. Es más, algunas constantes empíricas pueden ser calculadas y los valores obtenidos son cercanos a las cantidades recomendadas.

\footnotetext{
${ }^{*}$ This work was supported by RFBI (project 01-01-00783).

${ }^{\dagger}$ Institute of Computational Technologies, Russian Academy of Science, Novosibirsk, Russia. E-mail: vova@lchd.ict.nsc.ru.

${ }^{\ddagger}$ Institute of Computational Technologies, Russian Academy of Science, Novosibirsk, Russia. E-mail: chernykh@ict.nsc.ru.
} 
Palabras clave: aproximación de equilibrio local, estela turbulenta, método de restricciones diferenciales, variedades invariantes, soluciones auto-similares.

Mathematics Subject Classification: 76F02, 76F55, 76F60, 35Q35, 58J70.

\section{Introduction}

The method of differential constraints advanced by Cartan and Yanenko is of a special interest in view of its application to Parametric Turbulent Models. A concept of algebraic expressions for the $n$-order moments of statistical characteristics of turbulent flow as the equations of invariant manifolds of the corresponding transport equations enables us to examine the closure procedure for momentum equations. The invariant manifolds were used in [1], [2] for investigating the problem of the development of a shearless mixing layer in the third-order closure model wherein we showed that this approach may be used to obtain new reductions of the models under consideration and to construct a class of explicit solutions. Due to the differential constraints derived, it was established that the equation of the invariant manifold (differential constraints of the model) coincides with the classical tensor-invariant Hanjalic-Launder algebraic model of the triple correlations for an unstratified flow and with the Zeman-Lumley model for a stratified flow.

The mathematical tools necessary for the expression of the above-mentioned concept are provided by Symmetry Analysis [3]. We begin with a preliminary discussion and basic notions.

\subsection{Differential constraints and algebraic parameterizations of higher moments}

We recall that the statistical moments method of turbulent flows structure description is based on representation of sought quantities as a sum of mean value and turbulent fluctuations, as suggested by Osborne Reynolds. It is well-known that using NavierStokes equations and the averaging process, we can obtain the transport equations for moments of arbitrary order but these equations contain turbulence correlations of the next higher order. Therefore, closure of equations cannot be obtained by resorting to equations for moments of higher and higher order; an exact turbulence model contains infinite number of the transport equations and includes the infinite chain of FriedmannKeller equations [4]. Conversion of this infinite chain of the transport equations to a certain closed set of equations is the so-called closure problem for momentum equations which appears in Parametric Turbulent Models. Closed mathematical models are obtained by an approximation of the correlations of a certain order in terms of lower order correlations and mean-flow quantities. Analysis of those models shows that the closure procedures for the infinite chain of momentum equations are based (often implicitly) on the assumption that the system under consideration of differential equations admit invariant manifolds (or sets). In fact, the derivation of the closure relationships (algebraic parameterizations of higher moments) attracts various proposals (or hypotheses) for modeling the turbulence,

for example, the eddy-viscosity concept, eddy-diffusivity concept and others. As is pointed 
out by Chorin in [5], closure relations are, as a rule, derived using empirical hypotheses and certain assumptions, which are often poorly justified. In approximate equilibrium shear flow it is usually supposed that both the production and the dissipative terms dominate in the kinetic turbulent equation. With the above-mentioned model assumption, the closed form of the corresponding model is obtained by the algebraic relationship between the mean-velocity gradient and the tangential Reynolds stress. The parameterization of this type cannot explain and describe the effects of nonlocal phenomenon of turbulent transport. To overcame this deficiency under such modeling, it is used the technique based on constructing approximate algebraic parameterizations of higher order moments. It is supposed that the problems which are not solved by means of the $n$-order models can be solved by the $(n+1)$-order model of turbulence. For example, the triple correlations of velocity field can be expressed by the first- and second-order moments in an algebraic form. This law was employed in different forms, and an important step in the development is to give up the direct link in the gradient-type form. As a rule, Hanjalic-Launder (ZemanLumley) algebraic relationship for unstratified (stratified) flows is obtained by the triple correlations equation in ignoring the convective and the gradient production terms.

In the framework of the theory of overdetermined systems, a checking procedure for correct change of a differential equation by corresponding closed relationship consists in examining on compatibility of an overdetermined system (the $n$ th-order closure model) with adjoint differential constraint (i.e., with the algebraic relationship for corresponding momentum).

The method of differential constraints [6] provides the general approach to investigating overdetermined systems. This approach can be used to justify algebraic models applied to the calculation of the statistical moments. In practice, the classical methods (from the theory of overdetermined systems) for studying overdetermined systems may be difficult, because it is necessary to find solutions to overdetermined systems using the Requeir theory.

The notion of invariant manifolds for a system of partial differential equations of a very general form (an extension of the invariant relations introduced by Levi-Chevita and Amaldy) allows us to find certain classes of differential constraints. In [7] the method of local determining equations of a system of evolution equations was proposed which generalizes the defining equations of the symmetry groups and makes it possible to find invariant manifolds admissible by a system and then, to obtain explicit solutions of a system.

The main aim of the present article is to analyze the local-equilibrium approximations in the problem of a far plane turbulent wake. Local-equilibrium approximations of secondorder moments are used while modeling turbulent flows. We show that the use of localequilibrium approximation for the second-order Hanjalic-Launder model [9] of a far plane turbulent wake dynamics is associated with a vanishing of the Poisson bracket for the defect of the averaged longitudinal velocity component $U_{1}$ and of the kinetic energy of turbulence $e$. The result obtained may be considered as the criterion for correct using the local-equilibrium approximations in modeling shear flows. The numerical calculations demonstrate realizability of the criterion obtained for turbulent flows. Numerical modeling is performed with the use of $\left(e, \epsilon,\left\langle u^{\prime} v^{\prime}\right\rangle\right)$-model of turbulence [11] and the initial data 
compatible with experiment.

Moreover, we also analyze the local-equilibrium approximations of the third-order moments based on the above-mentioned concept in the problem of the dynamics of a momentumless turbulent wake. Reduction of the models under consideration on the invariant manifolds obtained made it possible to find self-similar solutions to the problem and to separate a class of particular solutions.

\subsection{Invariant manifolds of evolution equations}

Let us briefly present the special terminology of Symmetry Analysis (see [3], [7] for more details).

We recall some notions. It is well-known that a dynamical system of ordinary differential equations

$$
x_{t}^{i}=f^{i}(\vec{x}), \quad i=1, \ldots, n, \quad \vec{x}=\left(x^{1}, \ldots, x^{n}\right)
$$

generates local one-parametric group $G_{1}$ with the vector field

$$
V_{f}=f^{1} \frac{\partial}{\partial x^{1}}+\cdots+f^{n} \frac{\partial}{\partial x^{n}} .
$$

A regular manifold $M$ given by the equations

$$
g^{1}(\vec{x})=\cdots=g^{s}(\vec{x})=0, \quad s<n,
$$

is called the invariant manifold under the group $G_{1}$ if

$$
\left.V_{f}\left(g^{i}\right)\right|_{M}=0, \quad 1 \leq i \leq s .
$$

Invariant sets of the above dynamical system is invariant manifolds of the corresponding one-parametric group $G_{1}$.

Let us consider a system of evolution equations $\mathcal{F}$

$$
u_{t}^{i}=\mathcal{F}^{i}\left(t, x_{1}, \ldots, x_{n}, u^{1}, \ldots, u_{\lambda}^{k}, \ldots,\right)
$$

where $i=1, \ldots, m, u_{\lambda}^{k}=\partial^{\lambda} u^{k} / \partial x_{1}^{\lambda_{1}} \ldots \partial x_{n}^{\lambda_{n}}$.

A set(manifold) $\mathcal{H}$ given by equations

$$
h_{i}\left(t, x_{1}, \ldots, x_{n}, \ldots, u^{1}, \ldots, u^{m}, \ldots, u_{\lambda}^{k}, \ldots\right)=0
$$

is said to be the invariant set (manifold) of system $\mathcal{F}$ if

$$
\begin{gathered}
\left.V_{\mathcal{F}}\left(h^{i}\right)\right|_{[\mathcal{H}]_{0}}=0 \\
V_{\mathcal{F}}=\frac{\partial}{\partial t}+\sum_{i=1}^{m} \mathcal{F}^{i} \frac{\partial}{\partial u^{1}}+\sum_{i=1}^{m} D^{\alpha}\left(\mathcal{F}^{i}\right) \frac{\partial}{\partial u_{\alpha}^{i}}
\end{gathered}
$$

where $\alpha=\left(\alpha_{1}, \ldots, \alpha_{n}\right), D^{\alpha}=D_{x_{1}}^{\alpha_{1}} \ldots D_{x_{n}}^{\alpha_{n}}$. 
The invariant condition can be written in the following equivalent form

$$
\left.\left.D_{t}\left(h_{i}\right)\right|_{[\mathcal{F}]_{0}}\right|_{[\mathcal{H}]_{0}}=0
$$

Here $[\mathcal{F}]_{0}$ is $\infty$-prolongation (see $[7]$ ) of $\mathcal{F}$ with respect to $x_{1}, \ldots, x_{n}$. The set $[\mathcal{H}]_{0}$ is determined by analogy.

In an application of invariant relations between the system $\mathcal{F}$ and set (manifold) $\mathcal{H}$ we demonstrate the following useful

Theorem 1.1 [7] Assume that the system $\mathcal{F}$ has an invariant set(manifold) of the form $\mathcal{H}$ which is solved with respect to higher derivatives and the initial conditions are given by $u_{n_{i}}^{i}\left(x_{0}, t_{0}\right)=c_{i k_{i}}, c_{i k_{i}} \in \mathbb{R}$, then in some neighborhood of $\left(x_{0}, t_{0}\right) \in \mathbb{R}^{2}$ there exists a unique smooth solution of $\mathcal{F}$.

\section{Hanjalic-Launder model for a far plane turbulent wake dynamics}

In shear flows the turbulence is said to be in the state of local-equilibrium when the production of the kinetic energy of a turbulent motion is equal to the viscous dissipation $(P=\epsilon)$. It is well known that at most cases the production $P$ and the viscous dissipation $\epsilon$ are not balanced in all field of the flow, although these quantities are a sufficiently close to each other with respect to exponents of quantities. Moreover, the local-equilibrium state partially has the nature of a hypothesis. Nevertheless, this approximation is realized in the so-called layer of constant stress of turbulent boundary layer. In practical calculations, the approach based on using local-equilibrium approximations is widely employed in mathematical modeling of thin shear layers and, as an example, the dynamics of a far plane turbulent wake are investigated.

The aim of this section is to give an analysis of local-equilibrium approximations of second-order moments which are used in modeling turbulent flows (see [10]) by the method of differential constraints.

Two mathematical models are used to describe the flow in a far plane turbulent wake. Model 1 is based on the classical $(e, \epsilon)$-model (see [11]):

$$
\begin{gathered}
U_{0} \frac{\partial U_{1}}{\partial x}=\frac{\partial}{\partial y}\left\langle u^{\prime} v^{\prime}\right\rangle, \\
U_{0} \frac{\partial e}{\partial x}=\frac{\partial}{\partial y} \nu_{t_{1}} \frac{\partial e}{\partial y}+P-\epsilon, \\
U_{0} \frac{\partial \epsilon}{\partial x}=\frac{\partial}{\partial y} \nu_{t_{2}} \frac{\partial \epsilon}{\partial y}+\frac{\epsilon}{e}\left(C_{\epsilon_{1}} P-C_{\epsilon_{2}} \epsilon\right) .
\end{gathered}
$$

Here $U_{0}$ is the velocity of a nonperturbed flow; $U_{1}=U_{0}-U$ denotes the defect of the averaged longitudinal velocity component $U_{1}$; the angle brackets $\langle\cdot\rangle$ is the symbol of averaging; $\nu_{t_{1}}, \nu_{t_{2}}$ are the turbulent viscosity coefficients;

$$
\nu_{t_{1}}=C_{\mu} \frac{e^{2}}{\epsilon}, \quad \nu_{t_{2}}=\frac{\nu_{t_{1}}}{\sigma_{\epsilon}}
$$


$e$ is the kinetic energy turbulence, $\epsilon$ is the rate of dissipation of kinetic energy into heat; and

$$
P=\left\langle u^{\prime} v^{\prime}\right\rangle \frac{\partial U_{1}}{\partial y}
$$

is the production of the kinetic energy of turbulence due to the gradients of the averaged velocity, where

$$
\left\langle u^{\prime} v^{\prime}\right\rangle=C_{\mu} \frac{e^{2}}{\epsilon} \frac{\partial U_{1}}{\partial y} \equiv C_{\mu} e \hat{\tau} \frac{\partial U_{1}}{\partial y}
$$

with $\hat{\tau}=e / \epsilon$. In Model 2, the tangential Reynolds stress $\left\langle u^{\prime} v^{\prime}\right\rangle$ is determined from the differential equation (see [9])

$$
U_{0} \frac{\partial\left\langle u^{\prime} v^{\prime}\right\rangle}{\partial x}=\frac{\partial}{\partial y} \nu_{t_{3}} \frac{\partial\left\langle u^{\prime} v^{\prime}\right\rangle}{\partial y}-C_{\phi_{1}}\left\langle u^{\prime} v^{\prime}\right\rangle \frac{\epsilon}{e}+C_{\phi_{2}} e \frac{\partial U_{1}}{\partial y}
$$

where $\nu_{t_{3}}=C_{s} C_{\mu}^{-1} \nu_{t_{1}}$. Thus, Model 2 includes equations (1)-(3) and (6). The formula (5) is a consequence of employing the local-equilibrium approximation to equation (6). The quantities $\sigma_{\epsilon}, C_{\epsilon_{1}}, C_{\epsilon_{2}}, C_{\mu}, C_{\phi_{1}}$ and $C_{\phi_{2}}, C_{s}$ are empirical constant $\left(C_{\phi_{2}}=C_{\phi_{1}} C_{\mu}\right)$. The problem variables can be made dimensionless by using the characteristic length $D$, the body diameter and the velocity scale $U_{0}$.

\subsection{Invariant manifold of Model 2}

The algebraic model of local-equilibrium approximation (5) is derived from the closed equations of Model 2 by assuming that terms describing convective and diffusive transfer in (6) are negligible. The correct choice of an operator dominating in an equation at a certain stage of flow development requires the estimation of the joint contribution from all terms of the equation and must be justified (or refuted) by a certain formal procedure based only on the equations of the model without invoking any physical hypothesis. This derivation we present in the framework of the above-mentioned concept.

We consider the set $\mathcal{D}$ :

$$
\mathcal{D}=\left\{e, \hat{\tau}, U,\left\langle u^{\prime} v^{\prime}\right\rangle: \mathcal{G}^{1}\left(e, \hat{\tau}, U_{1},\left\langle u^{\prime} v^{\prime}\right\rangle\right) \equiv\left\langle u^{\prime} v^{\prime}\right\rangle-C_{\mu} \hat{\tau} e \frac{\partial U_{1}}{\partial y}=0\right\} .
$$

Derivation of formula (5) can be related to the invariance of the set $\mathcal{D}$ with respect to the flow generated by system (1)-(3), (6).

Together with equations (1)-(3) and (6) which govern the propagation of a turbulent perturbation in a fluid behind a body, the equation for $\hat{\tau}$ plays a crucial role for studying properties of system (1)-(3), (6). This equation is derived from equations (2),(3)and can be written in the form:

$$
\begin{gathered}
\frac{\partial \hat{\tau}}{\partial x}=\frac{1}{\epsilon} \frac{\partial e}{\partial x}-\frac{e}{\epsilon^{2}} \frac{\partial \epsilon}{\partial x}= \\
U_{0}^{-1}\left\{\frac{1}{\epsilon} \frac{\partial}{\partial y} \nu_{t_{1}} \frac{\partial e}{\partial y}+\frac{P}{\epsilon}-1-\frac{e}{\epsilon^{2}} \frac{\partial}{\partial y} \nu_{t_{2}} \frac{\partial \epsilon}{\partial y}+\frac{1}{\epsilon}\left(C_{\epsilon_{1}} P-C_{\epsilon_{2}} \epsilon\right)\right\}= \\
U_{0}^{-1}\left\{\frac{1}{\epsilon} \frac{\partial}{\partial y} C_{\mu} \hat{\tau}^{2} e \frac{\partial \epsilon}{\partial y}+\frac{1}{\epsilon} \frac{\partial}{\partial y} C_{\mu} \hat{\tau} e \epsilon \frac{\partial \hat{\tau}}{\partial y}+\frac{P}{\epsilon}-1-\right.
\end{gathered}
$$




$$
\left.\frac{1}{\epsilon} \hat{\tau} \frac{\partial}{\partial y} \frac{C_{\mu}}{\sigma_{\epsilon}} \hat{\tau} e \frac{\partial \epsilon}{\partial y}-\frac{1}{\epsilon} C_{\epsilon_{1}} P+C_{\epsilon_{2}}\right\} .
$$

At $\sigma_{\epsilon}=1$ (this value is recommended in [11]) and $C_{\epsilon_{1}}=1$ (the recommended value equals 1.4), the equation has the solution $\hat{\tau}(x, y) \equiv \hat{\tau}_{h}(x)=U_{0}^{-1}\left(C_{\epsilon_{2}}-1\right)\left(x+x_{0}\right)$. The theorem below provides the criterion of the invariance of $\mathcal{D}$ :

Theorem 2.1 Let $\left(U_{1}, e, \epsilon,\left\langle u^{\prime} v^{\prime}\right\rangle\right)$ be a sufficiently smooth solution to system (1)-(3), (6), and let $\sigma_{\epsilon}=C_{\epsilon_{1}}=1$. Assume that $C_{\phi_{2}}-C_{\phi_{1}} C_{\mu}=C_{\mu}\left(C_{\epsilon_{2}}-1\right)$. Then, the set $\mathcal{D}$ is the invariant manifold of system (1)-(3), (6) at $\hat{\tau}=\hat{\tau}_{h}$ if and only if the Poisson bracket is $\left\{e, U_{1}\right\}=0$.

The proof is based on direct calculation of the derivative $\partial / \partial x \mathcal{G}^{1}$ which leads us (taking into account (6)) to the formula

$$
\frac{\partial}{\partial x} \mathcal{G}^{1}=-e \frac{\partial U_{1}}{\partial y}\left(\hat{\tau}_{h x} U_{0} C_{\mu}+C_{\phi_{1}} C_{\mu}-C_{\phi_{2}}\right)-\hat{\tau}_{h} U_{0} C_{\mu}\left[\frac{\partial e}{\partial x} \frac{\partial U_{1}}{\partial y}-\frac{\partial e}{\partial y} \frac{\partial U_{1}}{\partial x}\right] .
$$

Using the relationships between the model constants and the form of the function $\hat{\tau}_{h}$, this formula can be written as (the expression in parentheses equals zero)

$$
\frac{\partial}{\partial x} \mathcal{G}^{1}=-U_{0} C_{\mu} \hat{\tau}_{h}\left\{e, U_{1}\right\}
$$

this completes the proof of the theorem.

Remark 2.1 Using the recommended values of the constants $C_{\phi_{1}}, C_{\mu}, C_{\epsilon_{2}}$ from [9], and calculating the right-hand of $C_{\phi_{2}}-C_{\phi_{1}} C_{\mu}=C_{\mu}\left(C_{\epsilon_{2}}-1\right)$ which equals 0.081, we find that $C_{\phi_{2}}$ is close to $C_{\phi_{1}} C_{\mu}=0.252$. Therefore, the value obtained $C_{\phi_{2}}$ differs only slightly from $C_{\phi_{2}}=C_{\phi_{1}} C_{\mu}$ recommended in [11], [9].

The simple example of flows where the Poisson bracket is $\left\{e, U_{1}\right\}=0$ are as follows: (1) a shearless flow with zero defect of the averaged longitudinal velocity component $\left(U_{1}=0\right)$; (2) - a flow with the degenerate components $e_{x}=U_{1 x}=0$; a less trivial example (3) - a flow where the production equals the viscous dissipation $P=\epsilon$.

\subsection{Computational results}

To illustrate the above statement, we have carried out a series of numerical experiments by using Models 1 and 2 with the initial conditions are given at the distance $x_{0} / D=625$. Initial conditions are selected in accordance to the experimental data of [13] for degenerate far plane turbulent wakes behind a cylinder. The initial distribution of the tangential stress $\left\langle u^{\prime} v^{\prime}\right\rangle$ is specified by formula (5). A finite difference algorithm for calculating, its test and realization, and the results of its application to the problem of turbulent wake dynamics were detailed in [19]. The computations are performed to double accuracy. Table 1 contains presents the quantity

$$
\delta^{\tilde{n}}=\frac{\max _{j}\left|\left(U_{1 x}^{h} e_{y}^{h}\right)_{j}^{\tilde{n}}-\left(U_{1 y}^{h} e_{x}^{h}\right)_{j}^{\tilde{n}}\right|}{\max _{j}\left(\left|\nabla^{h} e\right|_{j},\left|\nabla^{h} U_{1}\right|_{j}\right)} .
$$


which is considered as a function of the distance from the and, at $x=x^{\tilde{n}}$, characterizes the mesh analog of the Poisson bracket. Here $\left(U_{1 x}^{h}\right)_{j},\left(U_{1 y}^{h}\right)_{j},\left(e_{x}^{h}\right)_{j},\left(e_{y}^{h}\right)_{j},\left(\nabla^{h} e\right)_{j},\left(\nabla^{h} U_{1}\right)_{j}$ are the finite-difference approximations of the first derivatives and gradients at the node $\left.y=y_{j}, j=1, \ldots, N_{y}-1\right)$. Column 1 specifies the distance from the body $x / D$, and columns II, III, IV presents $\delta^{\tilde{n}}$ in the uniform meshes $1-3$ with the parameters $h_{x}=$ $0.5, h_{y}=0.1 ; h_{x}=0.25, h_{y}=0.05 ;$ and $h_{x}=0.125, h_{y}=0.025$, respectively.

The calculations are based on Model 2 and indicate that the mesh analog of the Poisson bracket is close to zero for all mesh parameters.

Tables 2 and 3 present the axial values calculated in Models 1 and 2, respectively, for the turbulence energy $e_{0}=e_{0}(x)=e(x, 0)$, the rate of dissipation $\epsilon_{0}=\epsilon_{0}(x)=\epsilon(x, 0)$ and the defect of the averaged longitudinal velocity component $U_{d 0}=U_{1}(x, 0)$.

According to these data, which were obtained with the mesh parameters $h_{x}=0.25$, $h_{y}=0.05$, wake parameters calculated in Models 1 and 2 close to each other. The difference between the corresponding arrays of $U_{1}, e$ and $\epsilon$ is no more that the difference between the respective axial values.

Thus, the above theorem and the calculations show that the approximation of local equilibrium is applicable for determining the tangential Reynolds stress $\left\langle u^{\prime} v^{\prime}\right\rangle$ in the problem of dynamics of a far plane turbulent wake.

\begin{tabular}{c|ccc}
\hline \hline I & II & III & IV \\
\hline 725 & $0.19 \times 10^{-6}$ & $0.19 \times 10^{-6}$ & $0.19 \times 10^{-6}$ \\
925 & $0.11 \times 10^{-6}$ & $0.11 \times 10^{-6}$ & $0.11 \times 10^{-6}$ \\
1125 & $0.66 \times 10^{-7}$ & $0.65 \times 10^{-7}$ & $0.65 \times 10^{-7}$ \\
1425 & $0.35 \times 10^{-7}$ & $0.32 \times 10^{-7}$ & $0.32 \times 10^{-7}$ \\
\hline \hline
\end{tabular}

Table 1: Variation of $\delta^{\tilde{n}}$ as a function of the distance from the body.

\begin{tabular}{c|ccc}
\hline \hline $\mathrm{x} / \mathrm{D}$ & $e_{0}{ }^{(1)}$ & $\varepsilon_{0}{ }^{(1)}$ & $U_{d 0}^{(1)}$ \\
\hline 725 & $0.145 \times 10^{-3}$ & $0.253 \times 10^{-6}$ & $0.357 \times 10^{-1}$ \\
925 & $0.112 \times 10^{-3}$ & $0.148 \times 10^{-6}$ & $0.335 \times 10^{-1}$ \\
1125 & $0.929 \times 10^{-4}$ & $0.992 \times 10^{-7}$ & $0.316 \times 10^{-1}$ \\
1425 & $0.749 \times 10^{-4}$ & $0.626 \times 10^{-7}$ & $0.294 \times 10^{-1}$ \\
\hline \hline
\end{tabular}

Table 2: Axial values of the turbulence energy, the rate of dissipation and the defect of the averaged longitudinal velocity component according to Model 1.

\section{Linearized Prandtl model for a far plane turbulent wake dynamics}

The aim of the present subsection is to find exactly (without using numerical experiments) a class of solutions to the problem under consideration which admits the use of the localequilibrium approximation. In other words, we obtain such solutions to the problem 


\begin{tabular}{c|ccc}
\hline \hline $\mathrm{x} / \mathrm{D}$ & $e_{0}{ }^{(2)}$ & $\varepsilon_{0}{ }^{(2)}$ & $U_{d 0}^{(2)}$ \\
\hline 725 & $0.145 \times 10^{-3}$ & $0.253 \times 10^{-6}$ & $0.358 \times 10^{-1}$ \\
925 & $0.113 \times 10^{-3}$ & $0.148 \times 10^{-6}$ & $0.335 \times 10^{-1}$ \\
1125 & $0.930 \times 10^{-4}$ & $0.996 \times 10^{-7}$ & $0.316 \times 10^{-1}$ \\
1425 & $0.747 \times 10^{-4}$ & $0.631 \times 10^{-7}$ & $0.294 \times 10^{-1}$ \\
\hline \hline
\end{tabular}

Table 3: The same as in Table 2, but according to Model 2.

that the tangential Reynolds stress $\left\langle u^{\prime} v^{\prime}\right\rangle$ is realized in the form of the local-equilibrium approximation.

We consider a simple model which enables us to express explicitly a class of such admissible solutions. The following linearized models [13] based on the Prandtl approximation of the turbulent viscosity are used: Model 3 includes the equation

$$
U_{0} \frac{\partial U_{1}}{\partial x}=\frac{\partial}{\partial y}\left\langle u^{\prime} v^{\prime}\right\rangle
$$

Equation (8) is not closed, and as usually, it is assumed that

$$
\left\langle u^{\prime} v^{\prime}\right\rangle=\nu_{T} \frac{\partial U_{1}}{\partial y}
$$

In addition to equation (8) we also use the turbulent energy transformation equation

$$
U_{0} \frac{\partial E}{\partial x}=\nu_{T} \frac{\partial^{2} E}{\partial y^{2}}+\frac{\partial U_{1}}{\partial y}\left\langle u^{\prime} v^{\prime}\right\rangle-C_{0} \nu_{T} \frac{E}{L^{2}} .
$$

In equations (8), (9) $C_{0}$ is an empirical constant, $\nu_{T}$ is the turbulent viscosity determined by the formula

$$
\nu_{T}=\chi U_{1 \max } L,
$$

where $\chi$ is an empirical constant, $U_{1 \max }(x)$ is the maximum value of $U_{1}$ for any fixed value of the variable $x, L=L(x)$ is the characteristic turbulence length scale.

Unknown functions in the problem are $U_{1}, E$ and the scale of turbulence $L$ which is taken to be

$$
L=\frac{1}{2}\left(L_{1}-L_{2}\right)
$$

where $L_{1}$ and $L_{2}$ are defined from the equalities $U_{1}\left(L_{1}(x), x\right)=\frac{1}{2} U_{1}\left(y_{\max }(x), x\right)$ for $y>y_{\max }(x)$ and $U_{1}\left(L_{2}(x), x\right)=\frac{1}{2} U_{1}\left(y_{\max }(x), x\right)$ for $y<y_{\max }(x)$. We denote here by $y_{\max }(x)$ the value of variable on which $\max _{y \in R} U_{1}(y, x)$ is attained.

In Model 4 the tangential Reynold stress $\left\langle u^{\prime} v^{\prime}\right\rangle$ is determined from the equation (see equation (6)):

$$
U_{0} \frac{\partial\left\langle u^{\prime} v^{\prime}\right\rangle}{\partial x}=\nu_{T} \frac{\partial^{2}}{\partial y^{2}}\left\langle u^{\prime} v^{\prime}\right\rangle-C_{\phi_{1}} C_{\mu}\left\langle u^{\prime} v^{\prime}\right\rangle \frac{E}{\nu_{T}}+C_{\phi_{2}} E \frac{\partial U_{1}}{\partial y}
$$




\subsection{Invariant manifold of Model 4}

We prove that equation (10) admits the invariant set

$$
\mathcal{D}_{L}=\left\{\nu_{T}, U_{1},\left\langle u^{\prime} v^{\prime}\right\rangle: \mathcal{G}_{L}^{1}\left(\nu_{T}, U_{1},\left\langle u^{\prime} v^{\prime}\right\rangle\right) \equiv\left\langle u^{\prime} v^{\prime}\right\rangle-\nu_{T} \frac{\partial U_{1}}{\partial y}=0\right\}
$$

for certain class of solutions to (8), (9) (10).

Theorem 3.1 Let $\left(U_{1}, E,\left\langle u^{\prime} v^{\prime}\right\rangle\right)$ be a sufficient smooth solution of (8), (9), (10). Assume that $C_{\phi_{2}}=C_{\phi_{1}} C_{\mu}$. Then the set $\mathcal{D}_{L}$ is the invariant manifold of system (8), (9) (10) if and only if $\partial \nu_{T} / \partial x=0$.

By calculating the derivative $\mathcal{D}_{L}$ with respect to $x$ we deduce that

$$
\frac{\partial}{\partial x} \mathcal{D}_{L}=-\left(C_{\phi_{2}}-C_{\phi_{1}} C_{\mu}\right) E \frac{\partial U_{1}}{\partial y}-\frac{\partial \nu_{T}}{\partial x} \frac{\partial U_{1}}{\partial y} .
$$

The proof is completed by taking into account the conditions of the theorem.

Remark 3.1 The equality $C_{\phi_{2}}=C_{\phi_{1}} C_{\mu}$ coincides with the recommended relationship between the empirical constants $C_{\phi_{2}}, C_{\phi_{1}}, C_{\mu}$, see [9], [11].

Let us show that the equality $\partial \nu_{T} / \partial x=0$ is realized for a self-similar solution to (8), (9), (10).

We look for a solution to Model 4 in the following form: $u=u_{a}(y, t), e=e_{a}(y, t)$, $w=w_{a}(y, t) \equiv \nu_{T a} \partial u_{a} / \partial y$ where $u(y, t)=U_{1}\left(y, \theta^{-1}(t)\right), e(y, t)=E\left(y, \theta^{-1}(t)\right)$, $w(y, t)=\left\langle u^{\prime} v^{\prime}\right\rangle\left(y, \theta^{-1}(t)\right), \nu_{T a}(t)=\chi u_{\max } l(t)$ and $l(t)=L\left(\theta^{-1}(t)\right)$; the sought functions $u_{a}(y, t), e_{a}(y, t), w_{a}(y, t)$ to be determined in self-similar variables. Here

$$
t=\theta(x) \equiv U_{0}^{-1} \int_{x_{0}}^{x} \nu_{T}(s) d s .
$$

Equations (8),(9) for $u_{a}(y, t), e_{a}(y, t), w_{a}(y, t)$ can be written as

$$
\begin{gathered}
u_{a t}=\frac{\partial^{2} u_{a}}{\partial y^{2}}, \quad(y, t) \in \mathbb{R} \times \mathbb{R}_{+}, \\
e_{a t}=\frac{\partial^{2} e_{a}}{\partial y^{2}}+\left(\frac{\partial u_{a}}{\partial y}\right)^{2}-C_{0} \frac{e_{a}}{l^{2}}, \quad(y, t) \in \mathbb{R} \times \mathbb{R}_{+} .
\end{gathered}
$$

The initial conditions for equations $(8),(9)$

$$
U_{1}\left(y, x_{0}\right)=U_{0}(y), \quad E\left(y, x_{0}\right)=E_{0}(y)
$$

are assumed to be positive bell-shaped functions such that $U_{0}(y) \rightarrow 0, E_{0}(y) \rightarrow 0$ as $y \rightarrow \pm \infty$. We set

$$
u_{a}(y, 0) \equiv u_{0}(y)=U_{0}(y), \quad e_{a}(y, 0) \equiv e_{0}(y)=E_{0}(y)
$$


Equation (12) has in $\mathbb{R} \times \mathbb{R}_{+}$the following well-known self-similar solution:

$$
u_{a}(y, t)=\frac{f_{a}(\xi)}{\sqrt{1+t}}, \quad \xi=\frac{y}{\sqrt{1+t}},
$$

where $f_{a}(\xi)=\frac{F_{0}}{2 \sqrt{\pi}} \exp \left(-\frac{\xi^{2}}{4}\right), \xi \in \mathbb{R}$. The solution $u_{a}$ is obtained when the initial function $u_{0} \equiv u_{a}(y, 0)=\frac{F_{0}}{2 \sqrt{\pi}} \exp \left(-\frac{\xi^{2}}{4}\right)$ where $F_{0}$ is a positive constant such that $F_{0}=\left|u_{a}(y, 0)\right|_{L^{1}(\mathbb{R})}$. Due to symmetry of $u_{a}$, the function $l=l(t)$ is defined by the equality $u_{a}(l(t), t)=\frac{1}{2} u_{a}(0, t)$. Then, we can find $l$ by means of $u_{a}$. By the form of solution $u_{a}$ we easily find $l$ which is denoted by $l_{a}$ :

$$
l_{a}(t)=2 \sqrt{\ln 2(1+t)} .
$$

Then, we seek a self-similar solution to equation (13) in the form

$$
e_{a}(y, t)=(1+t)^{\alpha} \theta_{a}(\xi)
$$

where $\alpha$ is a constant, and $\theta_{a}(\xi)>0$ is a differentiable function. Substituting (16),(17), and $u_{a y}=-\frac{F_{0}}{4 \sqrt{\pi}} \xi \exp \left(-\frac{\xi^{2}}{4}\right)(1+t)^{-1}$ into (13), we obtain the equation

$$
\begin{gathered}
\alpha(1+t)^{\alpha-1} \theta_{a}-\frac{1}{2}(1+t)^{\alpha-1} \xi \theta_{a \xi} \\
=(1+t)^{\alpha-1} \theta_{a \xi \xi}+\frac{F_{0}^{2}}{16 \pi} \xi^{2}(1+t)^{-2} \exp \left(-\frac{\xi^{2}}{2}\right)-\frac{C_{0}}{4 \ln 2}(1+t)^{\alpha-1} \theta_{a} .
\end{gathered}
$$

Hence, we have the following condition: $\alpha=-1$. Then the time factors in the equation can be canceled. Thus, we have

$$
A_{c}\left(\theta_{a}\right) \equiv \theta_{a \xi \xi}+\frac{1}{2} \xi \theta_{a \xi}+\left(1-\frac{C_{0}}{4 \ln 2}\right) \theta_{a}+\frac{F_{0}^{2}}{16 \pi} \xi^{2} \exp \left(-\frac{\xi^{2}}{2}\right)=0
$$

By symmetry, it is necessary to require that

$$
\theta_{a}^{\prime}(0)=0
$$

Taking into account the properties $e_{0}(y)$ at infinity, it is necessary that the following boundary condition to be satisfied for the energy profile:

$$
\theta_{a}(\infty)=0
$$

Thus, the problem of constructing the self-similar solution $e_{a}$ reduces to the problem (18)-(20). It is well-known that homogeneous equation (18) has solutions with different asymptotic behavior as $\xi \rightarrow \infty$. These solutions depend on the parameter $c$. Thus, we construct $\theta_{a}(\xi)$, separating the parameter $c$ which is supposed to be chosen so that the solution obtained satisfactory describes the known experiment data presented in [13]. 
To find such a solution we consider the family of boundary value problems for equation (18):

$$
A_{c}\left(\theta_{a}\right)=0, \quad \theta_{a}(0)=\mu, \quad \theta_{a}(\infty)=0,
$$

where $\mu$ is a positive parameter. We put

$$
\eta=\frac{\xi}{\sqrt{2}}, \quad C_{0}=6 \ln 2, \quad D \equiv \frac{F_{0}^{2}}{4 \mu \pi}, \quad h(\eta)=\frac{1}{\mu} \theta_{a}(\sqrt{2} \eta) .
$$

Then equation (18) takes the form

$$
h_{\eta \eta}+\eta h_{\eta}-h=-D \eta^{2} \exp \left(-\eta^{2}\right) .
$$

Its solution $h(\eta ; \mu)$ with allowance for the boundary conditions $h(0 ; \mu)=1, h(\infty ; \mu)=0)$ is

$$
\begin{aligned}
h(\eta ; \mu)= & \eta\left\{\frac{3}{2} D \sqrt{\pi}[1-\Phi(\eta \sqrt{2})]-\frac{(1+2 D) \sqrt{\pi}}{\sqrt{2}}[1-\Phi(\eta)]\right\} \\
& +(1+2 D) \exp \left(-\frac{\eta^{2}}{2}\right)-2 D \exp \left(-\eta^{2}\right),
\end{aligned}
$$

where $\Phi(z) \equiv \sqrt{\frac{2}{\pi}} \int_{0}^{z} \exp \left(-\frac{s^{2}}{2}\right) d s$. The solution $h(\eta ; \mu)$ defines the sought self-similar solution $\theta_{a}(\xi)$ for $\mu=(3 \sqrt{2}-4) F_{0}^{2} /(8 \pi)$ (in view of the condition (19) in accordance with the equality $h^{\prime}(0 ; \mu)=3 D(\sqrt{\pi} / 2)-(1+2 D)(\sqrt{\pi} / \sqrt{2})$ and $h(\eta ; \mu)$ is close to the experimental data [13].

Noteworthy is an interesting property of the function $h$ : it attains a maximum value at $\eta^{*}>0$. The found numerical value $C_{0}$ in the form $C_{0}=6 \ln 2$ is of importance for the analysis of the asymptotic stability.

It is ease to check that the viscous turbulent coefficient $\nu_{T a}$ is constant. Indeed, by the exact formulas for $u_{a}(y, t)$ and $l_{a}(t)$, we can write

$$
\nu_{T a}=\chi \frac{f_{a}(0)}{\sqrt{1+t}} 2 \sqrt{\ln 2} \sqrt{1+t} \equiv \text { const. }
$$

Therefore, applying Theorem 3.1 we obtain that

$$
\left\langle u^{\prime} v^{\prime}\right\rangle\left(y, \theta^{-1}(x)\right)=w_{a}(y, t)=\nu_{T a} \frac{\partial u_{a}(y, t)}{\partial y} \equiv \nu_{T a} \frac{1}{(1+t)} \frac{\partial f_{a}(\xi)}{\partial \xi}
$$

satisfies equation (10) identically.

Thus, we showed that the local-equilibrium approximation is realized for the self-similar solution $u_{a}(y, t), e_{a}(y, t), w_{a}(y, t)$ of Model 4 .

Remark 3.2 There exists a connection between the invariant set $\mathcal{D}_{\mathcal{L}}$ and the existence of steady-state solutions to a system constructed by the time depending scaling $(y, t) \rightarrow(\xi, s)$, 
$s=\ln (1+t)$ which transforms the system

$$
\begin{aligned}
u_{t} & =\nu_{T}^{-1} \frac{\partial^{2} w}{\partial y^{2}}, \quad w(y, t)=\left\langle u^{\prime} v^{\prime}\right\rangle\left(y, \theta^{-1}(t)\right) \\
e_{t} & =\frac{\partial^{2} e}{\partial y^{2}}+\left(\frac{\partial u}{\partial y}\right)^{2}-C_{0} \frac{e}{l^{2}} \\
w_{t} & =\frac{\partial^{2} w}{\partial y^{2}}-C_{\phi_{1}} C_{\mu} w \frac{e}{\nu_{T}^{2}}+C_{\phi_{2}} \frac{e}{\nu_{T}} \frac{\partial u}{\partial y}
\end{aligned}
$$

into a system such that its a steady-state solution is determined by the self-similar solution $u_{a}(y, t), e_{a}(y, t), w_{a}(y, t)$ of system (23)-(25). As a result, we obtain that there exists an invariant manifold associated with an equilibrium state of the transformed system which contains the steady-state solution $\left(f_{a}, \theta_{a}, \nu_{T a} \partial f_{a} / \partial \xi\right)$.

Remark 3.3 Existence (and uniqueness) of an analytical in $x$ solution of the Cauchy problem to Model 3 together with proving convergence of the solution obtained to the selfsimilar solution of Model 4 were established in [12].

\section{Momentumless plane turbulent wake dynamics}

The objective of this section is to examine an algebraic expression for the triple correlation $\left\langle v^{3}\right\rangle$ of the transverse intensity of turbulence which generally used in certain second-order closure models. The exposition is demonstrated on the base of the well-known problem of the dynamics of a momentumless far turbulent wake in a homogeneous fluid.

Momentumless turbulent wakes behind bodies were considered in a number of works. It has been shown (see, for example [18]) that momentumless turbulent wakes behave quite differently from the turbulent wakes with a nonzero excess impulse. In particular, the momentumless turbulent wake can be considered as the shearless turbulent flow. There exists a sufficiently large number of publications [15]-[20] (comprehensive references can be found therein) in which the results of experimental and theoretical investigations of the dynamics of momentumless turbulent wakes are discussed. The results of laboratory and numerical experiments demonstrate a faster decrease the defect of the longitudinal velocity component in comparison with a wake flow behind towed body. As a result, a turbulent wake, which is considered on a distance about 10 diameters behind a body, is the shearless flow practically. The latter implies that the velocity of a flow coincides with the incident stream velocity, the tangential Reynolds stresses equal to zero.

We begin the modeling study of a momentumless far turbulent wake using Model 5 based on the Millionshchikov's quasinormality hypothesis for the parameterization of diffusion processes for the triple correlation. Recall that according to this hypothesis, it is assumed that all cumulants of fourth- and higher-order are negligible small in comparison with the corresponding correlation functions. 


\subsection{The governing equations to Model 5}

To describe the flow in a momentumless plane turbulent wake the following third-order closure model (see [21]) based on the use Millionshchikov's hypothesis can be applied (Model 5):

$$
\begin{aligned}
U_{0} \frac{\partial\left\langle v^{2}\right\rangle}{\partial x} & +\frac{\partial\left\langle v^{3}\right\rangle}{\partial y}=-C_{\epsilon} \frac{\left\langle v^{2}\right\rangle}{\tau} \\
U_{0} \frac{\partial\left\langle v^{3}\right\rangle}{\partial x} & +3\left\langle v^{2}\right\rangle \frac{\partial\left\langle v^{2}\right\rangle}{\partial y}=-\frac{3}{2}\left(C_{\epsilon}+C_{\Phi_{1}}\right) \frac{\left\langle v^{2}\right\rangle}{\tau} \\
U_{0} \frac{\partial \epsilon}{\partial x} & =C_{d} \frac{\partial}{\partial y}\left(\tau\left\langle v^{2}\right\rangle \frac{\partial \epsilon}{\partial y}\right)-C_{\epsilon_{2}} \frac{\epsilon}{\tau}
\end{aligned}
$$

where $\tau=e / \epsilon, e=3 / 2\left\langle v^{2}\right\rangle$. The constants involved in the model with the lower case letters are denoted by $C_{* *}$. Here $e, \tau$ are the kinetic energy and the time scale of turbulence respectively and $\left\langle v^{2}\right\rangle$ denotes the one-point velocity correlation of the second-order.

Introduce the new variable $t=\theta(x) \equiv U_{0}^{-1} x$ and the function $\hat{\tau}=\left\langle v^{2}\right\rangle / \epsilon \equiv 2 / 3 \tau$. Using the new coordinates $(y, t)$ system $(26)-(27)$ can be rewritten in the form

$$
\begin{aligned}
\frac{\partial\left\langle\hat{v}^{2}\right\rangle}{\partial t} & =-\frac{\partial\left\langle\hat{v}^{3}\right\rangle}{\partial y}-\frac{2}{3} C_{\epsilon} \frac{\left\langle\hat{v}^{2}\right\rangle}{\hat{\tau}} \\
\frac{\partial\left\langle\hat{v}^{3}\right\rangle}{\partial t} & =-3\left\langle\hat{v}^{2}\right\rangle \frac{\partial\left\langle\hat{v}^{2}\right\rangle}{\partial y}-\left(C_{\epsilon}+C_{\Phi_{1}}\right) \frac{\left\langle\hat{v}^{3}\right\rangle}{\hat{\tau}} \\
\frac{\partial \hat{\epsilon}}{\partial t} & =\hat{C}_{d} \frac{\partial}{\partial y}\left(\frac{3}{2} \hat{\tau}\left\langle\hat{v}^{2}\right\rangle \frac{\partial \hat{\epsilon}}{\partial y}\right)-\frac{2}{3} C_{\epsilon_{2}} \frac{\hat{\epsilon}}{\hat{\tau}}
\end{aligned}
$$

where $C_{d}=C_{s} / \sigma_{\epsilon} \approx 0.2, C_{\epsilon} \approx 1, C_{\Phi_{1}} \approx 2, C_{\epsilon_{2}} \approx 1.9$. Here $\left\langle\hat{v}^{2}\right\rangle=\left\langle v^{2}\right\rangle\left(\theta^{-1}(t), y\right)$, $\left\langle\hat{v}^{3}\right\rangle=\left\langle v^{3}\right\rangle\left(\theta^{-1}(t), y\right)$ and $\hat{\epsilon}=\epsilon\left(\theta^{-1}(t), y\right)$.

In addition, we indicate the equation for $\hat{\tau}$ which can be obtained from (29), (31)

$$
\begin{aligned}
& \frac{\partial \hat{\tau}}{\partial t}=-\frac{\hat{\tau}}{\left\langle\hat{v}^{2}\right\rangle} {\left[\frac{\partial\left\langle\hat{v}^{3}\right\rangle}{\partial y}+\delta_{1} \hat{\tau}\left\langle\hat{v}^{2}\right\rangle \frac{\partial^{2}\left\langle\hat{v}^{2}\right\rangle}{\partial z^{2}}+\delta_{1} \hat{\tau}\left(\frac{\partial\left\langle\hat{v}^{2}\right\rangle}{\partial y}\right)^{2}\right]+} \\
& \delta_{1}\left\langle\hat{v}^{2}\right\rangle \hat{\tau} \frac{\partial^{2} \hat{\tau}}{\partial y^{2}}+2 \delta_{1} \hat{\tau} \frac{\partial \hat{\tau}}{\partial y} \frac{\partial\left\langle v^{2}\right\rangle}{\partial y}-\delta_{1}\left(\frac{\partial \hat{\tau}}{\partial y}\right)^{2}\left\langle\hat{v}^{2}\right\rangle+\frac{2}{3}\left(C_{\epsilon_{2}}-C_{\epsilon}\right),
\end{aligned}
$$

where $\delta_{1}=(3 / 2) C_{d}, \delta_{1} \approx 0.3$ (see, for example [21]).

\subsection{Invariant manifold of Model 5}

We analyze the terms which vanish in a homogeneous situation. The most important are diffusion terms and the most popular assumption is the generalized gradient diffusion hypothesis (Hanjalic, Launder, see [14]) that in connection with Model 5 can be written in the form

$$
\left\langle\hat{v}^{3}\right\rangle=-C_{s} \hat{\tau}\left\langle\hat{v}^{2}\right\rangle \frac{\partial\left\langle\hat{v}^{2}\right\rangle}{\partial y}
$$


¿From physical point of view, the way of derivation of the algebraic relationship for the triple correlation was presented by Hanjalic and Launder [14] in the cases of axisymmetric and plane jets.

The aim of this subsection is to clarify when the set

$$
\mathcal{O}=\left\{\left\langle\hat{v}^{2}\right\rangle,\left\langle\hat{v}^{3}\right\rangle, \hat{\tau}: \mathcal{H}^{1}\left(\left\langle\hat{v}^{2}\right\rangle,\left\langle\hat{v}^{3}\right\rangle, \hat{\tau}\right) \equiv\left\langle\hat{v}^{3}\right\rangle+C_{s} \hat{\tau}\left\langle\hat{v}^{2}\right\rangle \frac{\partial\left\langle\hat{v}^{2}\right\rangle}{\partial y}=0\right\}
$$

is invariant under the flow generated by equation (30). At first, we present the criterion of invariance $\mathcal{O}$ in a general form and then, find a class of solutions to system (29)-(31) for which this criterion is fulfilled.

Theorem 4.1 Let $\left\{\left(\left\langle\hat{v}^{2}\right\rangle,\left\langle\hat{v}^{3}\right\rangle, \epsilon\right)\right\}$ be a set of sufficiently smooth solution of (29)-(31). Then the set $\mathcal{O}$ is the invariant manifold of system (29)-(31) if and only if

$$
\begin{array}{r}
C_{s} \frac{\partial \hat{\tau}}{\partial t} \frac{\partial\left\langle\hat{v}^{2}\right\rangle}{\partial y}\left\langle\hat{v}^{2}\right\rangle+C_{s} \hat{\tau} \frac{\partial\left\langle\hat{v}^{2}\right\rangle}{\partial t} \frac{\partial\left\langle\hat{v}^{2}\right\rangle}{\partial y}+C_{s} \hat{\tau}\left\langle\hat{v}^{2}\right\rangle \frac{\partial^{2}\left\langle\hat{v}^{2}\right\rangle}{\partial t \partial y}= \\
3\left\langle\hat{v}^{2}\right\rangle \frac{\partial\left\langle\hat{v}^{2}\right\rangle}{\partial y}+\left(C_{\epsilon_{2}}+C_{\Phi_{1}}\right) \frac{\left\langle\hat{v}^{3}\right\rangle}{\hat{\tau}} .
\end{array}
$$

The proof of this theorem follows from the formula

$$
\begin{aligned}
\frac{\partial}{\partial t} \mathcal{H}^{1}=-3\left\langle\hat{v}^{2}\right\rangle \frac{\partial\left\langle\hat{v}^{2}\right\rangle}{\partial y}-\left(C_{\epsilon_{2}}+C_{\Phi_{1}}\right) \frac{\left\langle\hat{v}^{3}\right\rangle}{\hat{\tau}}+ & C_{s} \frac{\partial \hat{\tau}}{\partial t} \frac{\partial\left\langle\hat{v}^{2}\right\rangle}{\partial y}\left\langle\hat{v}^{2}\right\rangle+ \\
& C_{s} \hat{\tau} \frac{\partial\left\langle\hat{v}^{2}\right\rangle}{\partial t} \frac{\partial\left\langle\hat{v}^{2}\right\rangle}{\partial y}+C_{s} \hat{\tau}\left\langle\hat{v}^{2}\right\rangle \frac{\partial^{2}\left\langle\hat{v}^{2}\right\rangle}{\partial t \partial y} .
\end{aligned}
$$

The formula is obtained if we differentiate $\mathcal{H}^{1}$ with respect to $t$ and replace $\partial / \partial t\left\langle v^{3}\right\rangle$ by means of equation (30).

Let us show that equality (34) is realized for a self-similar solution of system (29)-(31). It is well-known that in the case of a nonstratified flow, the problem of the dynamics of a momentumless plane turbulent wake admits a parametric group of scale transformation that enables us to find a self-similar solution of the form

$$
\left\langle\hat{v}_{a}^{2}\right\rangle=\frac{f(\xi)}{\left(t+t_{0}\right)^{2 \mu}}, \quad\left\langle\hat{v}_{a}^{3}\right\rangle=\frac{g(\xi)}{\left(t+t_{0}\right)^{3 \mu}}, \quad \hat{\epsilon}_{a}=\frac{h(\xi)}{\left(t+t_{0}\right)^{3 \mu+\nu}}, \quad \xi=\frac{y}{L}, \quad L=\left(t+t_{0}\right)^{\nu}
$$

where $t_{0}>0$ is a parameter.

We look for a solution to (29)-(31) in the form

$$
\left\langle\hat{v}^{2}\right\rangle=\left\langle\hat{v}_{a}^{2}\right\rangle, \quad\left\langle\hat{v}^{3}\right\rangle=\left\langle\hat{v}_{a}^{3}\right\rangle \equiv-\delta_{1} \hat{\tau}_{h}\left\langle\hat{v}_{a}^{2}\right\rangle \frac{\partial\left\langle\hat{v}_{a}^{2}\right\rangle}{\partial y}, \quad \hat{\epsilon}=\hat{\epsilon}_{a}
$$

where $\hat{\tau}(t, y) \equiv \hat{\tau}_{h}(t, y)=(2 / 3)\left(C_{\epsilon_{2}}-C_{\epsilon}\right)\left(t+t_{0}\right)$ solves equation (32) written for the functions $\left\langle\hat{v}_{a}^{2}\right\rangle,\left\langle\hat{v}_{a}^{3}\right\rangle, \hat{\epsilon}_{a}$ (the expression in square brackets in (32) equals zero). Then, the 
original system has the form

$$
\begin{aligned}
& 2 \mu f+(1-\mu) \xi f_{\xi}-g_{\xi}-\frac{2}{3} C_{\epsilon} h=0, \\
& -3 f f_{\xi}-(1-\mu) \xi g_{\xi}-\left(C_{\epsilon}+C_{\Phi_{1}}\right) \frac{g h}{f}+3 \mu g=0, \\
& C_{\epsilon}\left(C_{\epsilon_{2}}-C_{\epsilon}\right)\left(f f_{\xi}\right)_{\xi}-(1-\mu) \xi h_{\xi}+(2 \mu+1) h-\frac{2}{3} C_{\epsilon_{2}} \frac{h^{2}}{f}=0 .
\end{aligned}
$$

A direct calculation yields that $\nu=1-\mu$ and $\mu=1$. The last equality guarantees that equation (38) is satisfied identically, due to relations (36), when $\left(C_{d} / 2\right)\left(C_{\epsilon_{2}}+C_{\Phi_{1}}\right)=1+C_{d}\left(C_{\epsilon_{2}}-C_{\epsilon}\right)$. Therefore, system (37)-(39) is transformed to the ordinary differential equation for the profile $f$ and the algebraic relationships for the profiles $g, h$ :

$$
\begin{aligned}
& C_{\epsilon}\left(C_{\epsilon_{2}}-C_{\epsilon}\right)\left(f f_{y}\right)_{y}+\left[2-\frac{C_{\epsilon}}{\left(C_{\epsilon_{2}}-C_{\epsilon}\right)}\right] f=0, \\
& g=-C_{\epsilon}\left(C_{\epsilon_{2}}-C_{\epsilon}\right) f f_{y}, \\
& h=\frac{3 f}{2\left(C_{\epsilon_{2}}-C_{\epsilon}\right)} .
\end{aligned}
$$

The sign of $\left[2-\left(C_{\epsilon}\right) /\left(C_{\epsilon_{2}}-C_{\epsilon}\right)\right]$ is determined by values of coefficients $C_{* *}$ which are experimentally known numbers.

The following aim is to find a solution to (40). The boundary value conditions for equation (40) is determined by the physical model, we have $f(y) \equiv 0$ outside of turbulent wake.

To solve this problem, we consider a family of solutions to (40) satisfying the conditions $f^{\prime}(0)=0$ (the symmetry condition of the profile $f$ ) and $f(0)=f_{0}>0$. The existence and uniqueness solution to this problem on $(0,+\infty)$ follows from [22]. The solution obtained guarantees the existence of a symmetrical function $f$ on $(-\infty,+\infty)$ which solves equation (40). The function $f$ is positive on an interval $\left(-y_{0},+y_{0}\right), 0<+y_{0}<\infty$ and $f\left( \pm y_{0}\right)=0$ where $\pm y_{0} \rightarrow 0$ as $f_{0} \rightarrow 0$. Therefore, the size of support of the function $\left\langle\hat{v}_{a}^{2}\right\rangle$ depends on the value $f_{0}$.

Remark 4.1 The solution obtained $\left\langle\hat{v}_{a}^{2}\right\rangle$ makes sense only on the set $\left(\mathbb{R} \times\left[t_{0}, T\right]\right) \backslash\left\{t= \pm y_{0}\right\}$ due to the fact that both $\left\langle\hat{v}_{a}^{2}\right\rangle_{y y}$ and $\left\langle\hat{v}_{a}^{2}\right\rangle\left\langle\hat{v}_{a}^{2}\right\rangle_{y}$ are discontinuous functions on the line $\left\{t= \pm y_{0}\right\}$. This means that $\left\langle\hat{v}_{a}^{2}\right\rangle$ is not classical (and even generalized) solution of the equation on the plane $\mathbb{R} \times\left[t_{0}, T\right]$ but solves the boundary value problem in $\left[-y_{0},+y_{0}\right] \times\left[t_{0}, T\right], t_{0}<T$ with zero boundary conditions.

Once we have determined $\left\langle\hat{v}_{a}^{2}\right\rangle$, we can find $\left\langle\hat{v}_{a}^{3}\right\rangle$ and $\hat{\epsilon}_{a}$. By means of a direct calculation, we show that condition (34) holds for the triple of functions $\left\langle\hat{v}_{a}^{2}\right\rangle,\left\langle\hat{v}_{a}^{3}\right\rangle, \hat{\epsilon}_{a}$ under suitable assumptions on the model constants. We have the following

Theorem 4.2 Let $\delta_{1}=C_{s}$ and $\left(C_{d} / 2\right)\left(C_{\epsilon_{2}}+C_{\Phi_{1}}\right)=1+C_{d}\left(C_{\epsilon_{2}}-C_{\epsilon}\right)$, then formula (34) holds for the functions $\left\langle\hat{v}_{a}^{2}\right\rangle,\left\langle\hat{v}_{a}^{3}\right\rangle, \hat{\epsilon}_{a}$. 
Indeed, substituting in (34) the function $\left\langle\hat{v}_{a}^{2}\right\rangle,\left\langle\hat{v}_{a}^{3}\right\rangle, \hat{\epsilon}_{a}$ and taking into account the relationships between the constants, we obtain that

$$
\frac{\partial}{\partial t} \mathcal{H}^{1}=\frac{3 C_{d}}{2}\left(C_{\epsilon_{2}}+C_{\Phi_{1}}\right) f f_{y}-3\left[1+C_{d}\left(C_{\epsilon_{2}}-C_{\epsilon}\right)\right] f f_{y}
$$

This completes the proof of the theorem.

Remark 4.2 The value obtained $\delta_{1} \approx 0.3$ is close to the recommend value $C_{s} \approx 0.25$ in formula (33).

Millionshchikov's quasinormality hypothesis is known to be defective in some cases that leads to physically contradictory results, see, [4]. For example, we obtained that the boundaries of the turbulent wake are the functions which independent of $t$. The latter circumstance is a consequence of the fact that the triple-correlation equation is of the firstorder without a dumping mechanism for triple correlations. The approach proposed in [23] allows us to overcome this obstacle; the technique also includes a physically reasonable way for constructing approximate algebraic parameterizations of higher moments. In the second part of this section, we present a third-order model of turbulence to describe correctly the dynamics of a momentumless plane turbulent wake.

\subsection{The governing equations to Model 6}

To obtain a closed model of turbulent transport that does not imply equality to zero of the fourth-order cumulants, the closure procedure is performed at the level of the fifth moments, i.e. it is assumed that the fifth-order cumulants are equal to zero. Therefore (see, [1], [24]), the following third-order mathematical model can be used Model 2:

$$
\begin{aligned}
U_{0} \frac{\partial\left\langle v^{2}\right\rangle}{\partial x} & =-\frac{\partial\left\langle v^{3}\right\rangle}{\partial y}-\frac{2}{3} \epsilon, \\
U_{0} \frac{\partial\left\langle v^{3}\right\rangle}{\partial x} & =-\frac{\partial\langle C\rangle}{\partial y}-3\left\langle v^{2}\right\rangle \frac{\partial\left\langle v^{2}\right\rangle}{\partial y}-C_{2}\left\langle v^{3}\right\rangle \frac{\epsilon}{e}, \\
U_{0} \frac{\partial \epsilon}{\partial x} & =C_{d} \frac{\partial}{\partial y}\left(\frac{e}{\epsilon}\left\langle v^{2}\right\rangle \frac{\partial \epsilon}{\partial y}\right)-C_{\epsilon_{2}} \frac{\epsilon^{2}}{e} .
\end{aligned}
$$

We use the preceding notions for the quantities $e=3 / 2\left\langle v^{2}\right\rangle, \epsilon,\left\langle v^{2}\right\rangle$ and $\left\langle v^{3}\right\rangle$; $C=\left\langle v^{4}\right\rangle-3\left\langle v^{2}\right\rangle^{2}$ is the fourth-order cumulant of the vertical velocity fluctuations. In equations (43)-(45) the empirical constants $C_{2}, C_{d}, C_{\epsilon_{2}}$ are known from the previous section. We complete the system by an algebraic parametrization for the fourth-order cumulant $C$ which can be written as

$$
C=C_{1} \frac{e}{\epsilon}\left[6\left\langle v^{3}\right\rangle \frac{\partial\left\langle v^{2}\right\rangle}{\partial y}+\left\langle v^{2}\right\rangle \frac{\partial\left\langle v^{3}\right\rangle}{\partial y}\right] .
$$

It is well-known that the contribution of the second term in the algebraic model for the cumulant $C$ is essential [24]. 
It is convenient to introduce the new variable $t=\theta(x) \equiv U_{0}^{-1} x$. Thus the governing equations in the new coordinate $(y, t)$ take the form:

$$
\begin{aligned}
\frac{\partial\left\langle\bar{v}^{2}\right\rangle}{\partial t} & =-\frac{\partial\left\langle\bar{v}^{3}\right\rangle}{\partial y}-\alpha \frac{\left\langle\bar{v}^{2}\right\rangle}{\bar{\tau}}, \\
\frac{\partial\left\langle\bar{v}^{3}\right\rangle}{\partial t} & =\frac{\partial}{\partial y}\left[\kappa \bar{\tau}\left\langle\bar{v}^{2}\right\rangle \frac{\partial\left\langle\bar{v}^{3}\right\rangle}{\partial y}\right]-3\left\langle\bar{v}^{2}\right\rangle \frac{\partial\left\langle\bar{v}^{2}\right\rangle}{\partial y}-\gamma \frac{\left\langle\bar{v}^{3}\right\rangle}{\bar{\tau}}, \\
\frac{\partial \bar{\epsilon}}{\partial t} & =\frac{\partial}{\partial y}\left[\delta \bar{\tau}\left\langle\bar{v}^{2}\right\rangle \frac{\partial \bar{\epsilon}}{\partial y}\right]-\varrho \frac{\bar{\epsilon}}{\bar{\tau}},
\end{aligned}
$$

where $\alpha=2 / 3$ and $\kappa, \gamma, \delta, \varrho$ are constants which can be determined by the empirical model constants; $\bar{\tau}=\left\langle\bar{v}^{2}\right\rangle / \bar{\epsilon}$. Here $\left\langle\bar{v}^{2}\right\rangle=\left\langle v^{2}\right\rangle\left(\theta^{-1}(t), y\right),\left\langle\bar{v}^{3}\right\rangle=\left\langle v^{3}\right\rangle\left(\theta^{-1}(t), y\right)$ and $\bar{\epsilon}=\epsilon\left(\theta^{-1}(t), y\right)$.

We can obtain a simplified model ("standard" second-order model of turbulence) if we employ the local-equilibrium approximation to equation (47), replacing the equation for $\left\langle\bar{v}^{3}\right\rangle$ by the gradient-type algebraic parameterization

$$
\left\langle\bar{v}^{3}\right\rangle=-\frac{3}{\gamma} \bar{\tau}\left\langle\bar{v}^{2}\right\rangle \frac{\partial\left\langle\bar{v}^{2}\right\rangle}{\partial y} .
$$

\subsection{Invariant manifold of Model 6}

Let us introduce into consideration the following set

$$
\mathcal{O}_{L}=\left\{\left\langle\bar{v}^{2}\right\rangle,\left\langle\bar{v}^{3}\right\rangle, \bar{\tau}: \mathcal{H}_{L}^{1}\left(\left\langle\bar{v}^{2}\right\rangle,\left\langle\bar{v}^{3}\right\rangle, \bar{\tau}\right) \equiv\left\langle\bar{v}^{3}\right\rangle+\frac{3}{\gamma} \bar{\tau}\left\langle\bar{v}^{2}\right\rangle \frac{\partial\left\langle\bar{v}^{2}\right\rangle}{\partial y}=0\right\}
$$

We show that system (46)-(48) admits an invariant manifold of the form (50). The equation for $\bar{\tau}$ will be again crucial for our study of invariant sets of the system. This equation is a consequence of equations $(46),(48)$. Indeed, calculating the time derivative for $\bar{\tau}$ and using equations (46),(48), we obtain

$$
\begin{aligned}
\frac{\partial \bar{\tau}}{\partial t}=-\frac{\bar{\tau}}{\left\langle\bar{v}^{3}\right\rangle}\left[\frac{\partial\left\langle\bar{v}^{3}\right\rangle}{\partial y}+\delta \bar{\tau}\left\langle\bar{v}^{2}\right\rangle \frac{\partial^{2}\left\langle\bar{v}^{2}\right\rangle}{\partial y^{2}}+\right. & \left.\delta \bar{\tau}\left(\frac{\partial\left\langle\bar{v}^{2}\right\rangle}{\partial y}\right)^{2}\right]+\delta\left\langle\bar{v}^{2}\right\rangle \bar{\tau} \frac{\partial^{2} \bar{\tau}}{\partial y^{2}} \\
& +2 \delta \bar{\tau} \frac{\partial \bar{\tau}}{\partial y} \frac{\partial\left\langle\bar{v}^{2}\right\rangle}{\partial y}-\delta\left(\frac{\partial \bar{\tau}}{\partial y}\right)^{2}\left\langle\bar{v}^{2}\right\rangle+\varrho-\alpha .
\end{aligned}
$$

As the first result we have:

Theorem 4.3 Let $\left\{\left(\left\langle\bar{v}^{2}\right\rangle,\left\langle\bar{v}^{3}\right\rangle, \bar{\epsilon}\right)\right\}$ be a set of sufficiently smooth solutions of (46)-(48) and $3 / \bar{c}_{2}=\delta$. Assume that $3 / \delta+2 \alpha-\gamma=\varrho-\alpha$ and $\kappa=\delta$. Then system (46)-(48) admits the invariant set $\mathcal{O}_{L}$ and its reduction on the set $\mathcal{O}_{L}$ is of the form:

$$
\begin{aligned}
& \left\langle\bar{v}^{2}\right\rangle=\hat{\tau} \bar{\epsilon} \\
& \left\langle\bar{v}^{3}\right\rangle=-\delta \hat{\tau}\left\langle\bar{v}^{2}\right\rangle \frac{\partial\left\langle\bar{v}^{2}\right\rangle}{\partial y}, \\
& \frac{\partial \bar{\epsilon}}{\partial t}=\frac{\partial}{\partial y}\left[\delta \bar{\tau}\left\langle\bar{v}^{2}\right\rangle \frac{\partial \bar{\epsilon}}{\partial y}\right]-\varrho \frac{\bar{\epsilon}}{\bar{\tau}},
\end{aligned}
$$


where the function $\bar{\tau}(y, t) \equiv \bar{\tau}(t)$ solves the ordinary differential equation

$$
\frac{d \bar{\tau}}{d t}=(\varrho-\alpha) \quad\left(\text { a version of equation for } \bar{\tau} \text { on the set } \mathcal{O}_{L}\right) .
$$

To show that $\mathcal{O}_{L}$ is invariant under the flow generated by equation (47), it is sufficient to check that

$$
\left\langle\bar{v}^{3}\right\rangle=-\delta \bar{\tau}\left\langle\bar{v}^{2}\right\rangle \frac{\partial\left\langle\bar{v}^{2}\right\rangle}{\partial y}
$$

satisfies identically equation (47). The equation for $\bar{\tau}(y, t) \equiv \bar{\tau}(t)$ can be rewritten in the form

$$
\frac{d \bar{\tau}}{d t}=(\varrho-\alpha)
$$

since the expression in square brackets (see, the equation for $\bar{\tau}$ ) equals zero due to the formula

$$
\left\langle\bar{v}^{3}\right\rangle=-\delta \bar{\tau}\left\langle\bar{v}^{2}\right\rangle \frac{\partial\left\langle\bar{v}^{2}\right\rangle}{\partial y}
$$

Now consider the equality

$$
\frac{\partial}{\partial t} \mathcal{H}_{L}^{1}=\frac{\partial\left\langle\hat{v}^{3}\right\rangle}{\partial t}+\delta \frac{\partial}{\partial t}\left[\bar{\tau}\left\langle\bar{v}^{2}\right\rangle \frac{\partial\left\langle\bar{v}^{3}\right\rangle}{\partial y}\right]
$$

Using equation (47) and taking into account that $\partial \hat{\tau} / \partial y=0$, we can rewrite formula (55) in the form

$$
\begin{aligned}
\frac{\partial}{\partial t} \mathcal{H}_{L}^{1}=\kappa \bar{\tau} \frac{\partial\left\langle\bar{v}^{2}\right\rangle}{\partial y} \frac{\partial\left\langle\bar{v}^{3}\right\rangle}{\partial y}+\kappa \bar{\tau}\left\langle\bar{v}^{2}\right\rangle \frac{\partial^{2}\left\langle\bar{v}^{3}\right\rangle}{\partial y^{2}}- & 3\left\langle\bar{v}^{2}\right\rangle \frac{\partial\left\langle\bar{v}^{2}\right\rangle}{\partial y} \\
& -\frac{\gamma}{\bar{\tau}} \mathcal{H}_{L}^{1}+\gamma \delta\left\langle\bar{v}^{2}\right\rangle \frac{\partial\left\langle\bar{v}^{2}\right\rangle}{\partial y}+\delta \frac{\partial \bar{\tau}}{\partial t} \frac{\partial\left\langle\bar{v}^{2}\right\rangle}{\partial y}\left\langle\bar{v}^{2}\right\rangle \\
- & \delta \bar{\tau} \frac{\partial\left\langle\bar{v}^{2}\right\rangle}{\partial y} \frac{\partial\left\langle\bar{v}^{3}\right\rangle}{\partial y}-2 \delta \alpha \frac{\partial\left\langle\bar{v}^{2}\right\rangle}{\partial y}\left\langle\bar{v}^{2}\right\rangle-\delta \bar{\tau} \frac{\partial\left\langle\bar{v}^{2}\right\rangle}{\partial y} \frac{\partial^{2}\left\langle\bar{v}^{3}\right\rangle}{\partial y^{2}} .
\end{aligned}
$$

As a result, we obtain

$$
\begin{aligned}
& \frac{\partial}{\partial t} \mathcal{H}_{L}^{1}=(\kappa-\delta) \bar{\tau} \frac{\partial\left\langle\bar{v}^{2}\right\rangle}{\partial y} \frac{\partial\left\langle\bar{v}^{3}\right\rangle}{\partial y}+(\kappa-\delta) \bar{\tau}\left\langle\bar{v}^{2}\right\rangle \frac{\partial^{2}\left\langle\bar{v}^{3}\right\rangle}{\partial y^{2}}+ \\
&\left\langle\bar{v}^{2}\right\rangle \frac{\partial\left\langle\bar{v}^{2}\right\rangle}{\partial y}\left(\delta \frac{\partial \bar{\tau}}{\partial t}-2 \alpha \delta+\gamma \delta-3\right)-\frac{\gamma}{\bar{\tau}} \mathcal{H}_{L}^{1} .
\end{aligned}
$$

It follows from the assumptions for model constants and the equation for $\bar{\tau}$ that

$$
\frac{\partial}{\partial t} \mathcal{H}_{L}^{1}=\frac{\gamma}{\bar{\tau}} \mathcal{H}_{L}^{1}
$$

Therefore

$$
\left.\mathcal{H}_{L}^{1}\right|_{t=t_{1}}=\left.\mathcal{H}_{L}^{1}\right|_{t=t_{0}} \exp \left(-\gamma \int_{t_{0}}^{t_{1}} \bar{\tau}^{-1} d s\right)
$$

This completes the proof of the theorem. 


\subsection{Solutions on invariant manifold}

Theorem 4.3 enables us to reduce (46)-(48) to the algebraic differential expressions (51)(54) which can be easier analyzed. Indeed, to find a solution of the above system, we look for a solution of equation (48) where $\bar{\tau}$ is determined by the formula $\bar{\tau}(t)=(\varrho-\alpha)\left(t+t_{0}\right)$. Once we have determined $\bar{\epsilon}$, we can find $\left\langle\bar{v}^{2}\right\rangle$ and $\left\langle\bar{v}^{3}\right\rangle$ by formulas (51),(52).

We put at $t=t_{0}$ the following physically correct condition:

$$
\bar{\epsilon}\left(y, t_{0}\right)=\bar{\epsilon}_{0}(y)
$$

where $\bar{\epsilon}_{0}(y)$ is a nonnegative "bell-shaped" function with a compact support. The functions $\left\langle\bar{v}^{2}\right\rangle\left(y, t_{0}\right)$ and $\left\langle\bar{v}^{3}\right\rangle\left(y, t_{0}\right)$ are determined by formulas $(51),(52)$.

Set

$$
\theta \equiv \theta(t)=\int_{0}^{t} \bar{\tau}^{2}(p) d p, \quad \varsigma(\theta)=\bar{\tau}\left(\theta^{-1}(t)\right), \quad \psi(\theta)=\frac{1}{\varsigma^{3}(\theta)}
$$

and

$$
\bar{\epsilon}(y, \theta)=u(y, \theta) \exp \left(-\int_{0}^{\theta} \psi(p) d p\right), \quad \text { where } \bar{\epsilon}(z, \theta)=\bar{\epsilon}(z, t) .
$$

The function $\theta(t)$ maps $[0,+\infty)$ onto $[0,+\infty)$ and for $u$ we have:

$$
\begin{gathered}
\frac{\partial u}{\partial \hat{\theta}}=\frac{\partial}{\partial y}\left[\delta u \frac{\partial u}{\partial y}\right], \quad \text { where } \hat{\theta}=\int_{0}^{\theta} \exp \left(-\int_{0}^{\xi} \psi(p) d p\right) d \xi, \\
u(y, 0)=\bar{\epsilon}_{0}(y) .
\end{gathered}
$$

It is easy to check that $\hat{\theta}:[0,+\infty) \rightarrow[0,+\infty)$. Therefore, in studying the Cauchy problem for equation (53), we can base our analysis on investigation of transformed problem (57), (58).

Remark 4.3 Equation (57) is usually called the porous medium equation. As it is wellknown, the porous medium equation appear in the description of a number of nonlinear processes in filtration, heat propagation, diffusion and so on. Nonnegative solutions are considered in the hole space for some time interval. The existence and uniqueness of weak solutions of this type is by now well understood (see, [22]). This equation exhibits a number of peculiar properties arising from its nonlinear and degenerate parabolic character. In particular, if an initial data have compact support, then the same happens with $u(\cdot, \hat{\theta})$ for every $\hat{\theta}>0$ and there appears a free boundary that separates the regions $u>0$ and $u=0$ that coincides in our problem with well-known experimental observations about finite speed of propagation of turbulent wake in an incompressible fluid. The behavior of the free boundary and of the solution near by the free boundary it is now well understood and can be explained in the form of jump discontinuities for the quantity $u_{y}$ and Rankine-Hugoniot conditions, see, [25] for a reference.

In contrast to previous Model 5 where the self-similar solution obtained was realized as a solution with separating variables in Model 6 the self-similar solution, which comes from differential equation (47) with diffusion operator for the triple correlation $\left\langle\hat{v}^{3}\right\rangle$, describes correctly the momentumless wake propagation from physics point of view. 
Self-similar solution to our problem is a solution of the form

$$
\left\langle\bar{v}_{a}^{2}\right\rangle=\frac{f(\xi)}{\left(t+t_{0}\right)^{2 \mu}}, \quad\left\langle\bar{v}_{a}^{3}\right\rangle=\frac{g(\xi)}{\left(t+t_{0}\right)^{3 \mu}}, \quad \bar{\epsilon}_{a}=\frac{h(\xi)}{\left(t+t_{0}\right)^{3 \mu+\nu}}, \quad \xi=\frac{y}{L}, \quad L=\left(t+t_{0}\right)^{\nu} .
$$

If we choose $\nu=1-\mu$, then the original system is transformed to the system of ordinary differential equations for the profiles $f, q$ and $h$ :

$$
\begin{aligned}
& 2 \mu f+(1-\mu) \xi f_{\xi}-g_{\xi}-\alpha h=0, \\
& \kappa\left(\frac{f^{2}}{h} g_{\xi}\right)_{\xi}-3 f f_{\xi}-(1-\mu) \xi g_{\xi}-\gamma \frac{g h}{f}+3 \mu g=0, \\
& \delta\left(\frac{f^{2}}{h} h_{\xi}\right)_{\xi}-(1-\mu) \xi h_{\xi}+(2 \mu+1) h-\varrho \frac{h^{2}}{f}=0 .
\end{aligned}
$$

The free similarity exponent $\mu$ has to be determined from a solution of the obtained nonlinear eigenvalue problem. This is a typical situation appearing in nonlinear diffusion problems where a conservation law does not exist. To find a solution to system (60)-(62), we use the existence of the invariant set obtained in Theorem 4.3. Setting that the model constants satisfy the equalities from Theorem 4.3 and $\mu=\varrho / 3(\varrho-\alpha)$, we obtain that system (60)-(62)) admits a reduction. As a result, we have the following boundary value problem for $h(\xi)$ :

$$
\begin{aligned}
& \delta\left(w_{c}^{2} h h_{\xi}+(1-\mu) \xi h_{\xi}\right)_{\xi}=0, \quad w_{c}=\varrho-\alpha, \\
& h(-\infty)=0, \quad h(+\infty)=0 .
\end{aligned}
$$

We note that equation (63) arises in the context of study self-similar solutions to the porous medium equation. As a result, we have that there exists a unique solution $h(\xi)$ to problem (63)-(64) which coincides with the well-known Barenblatt's solution [26].

Remark 4.4 Model 6 adequately reflects the behavior of some flow characteristics. The calculated values $\mu \approx 0.7, \nu \approx 0.3$ agree well with the Kolmogorov's law of decaying isotropic turbulent flow. These quantities also close to recommended values in [15].

\section{Conclusions}

It has been shown that the method of differential constraints is an effective tool to analyze parametric turbulent models for plane turbulent wakes that enables us to find new reduction of the models under consideration, and therefore to construct explicit solutions. An important application of the presented approach is obtaining the functional and algebraic relationships between various flow characteristics in exact form. Moreover, it is turned out that some empirical model constants may be calculated and their obtained values are sufficiently close to the standard quantities. It will avoid the need for an additional empirical information for the turbulent flow characteristics.

It should be noted that the use of the local-equilibrium approximation is associated with a vanishing of the Poisson bracket for the defect of the averaged longitudinal velocity component $U_{1}$ and the kinetic energy of turbulence $e$, its manifests the new effect for flow characteristics. 


\section{References}

[1] Grebenev, V.N.; Ilyushin, B.B.; Shokin, Yu.I. (2000) "The use of differential constraints for analyzing turbulence models", J. Nonl. Sci. Numer. Simulation 1(4): $305-317$.

[2] Grebenev, V.N.; Ilyushin, B.B. (2002) "Invariant sets and explicit solutions to a thirdorder model for the shearless stratified turbulent flow", J. Nonl. Math. Phys. 9(2): $74-86$.

[3] Ibragimov, N.H. (1985) Transformation Groups Applied to Mathematical Physics. Reidel.

[4] Monin, A.S.; Yaglom, A.M. (1994) Statistical Hydromechanics, Gidrometeoizdat Vol 1, 2, St.-Petersburg (in Russian).

[5] Chorin, A. (1977) Lecture Notices in Math. 615, Springer-Verlag, Berlin.

[6] Sidorov, A.F.; Shapeev, V.P.; Yanenko, N.N. (1984) The Differential Constraints Method and its Application to Gas Dynamics. Nauka, Novosibirsk (in Russian).

[7] Kaptsov, O.V. (1995) "B-determining equations: applications to nonlinear partial differential equations", Euro. J. Appl. Math. 6: 265-286.

[8] Grebenev, V.N.; Demenkov, A. G.; Chernykh, G.G. (2002) "Analysis of the localequilibrium approximation in the problem of a far plane turbulent wake", Doklady Physics of Russian Academy of Sciences 385(1): 57-60.

[9] Hanjalic, K.; Launder, B.F. (1972) "Fully developed asymetric flow in a plane channel", J. Fluid Mech. 51: 301-335.

[10] Lewellen, W. (1977) In: Handbook of Turbulence. Fundamentals and Applications. Plenum Press.

[11] Harsha, P. In: Handbook of Turbulence. Fundamentals and Applications. Plenum Press, 1977.

[12] Grebenev, V.N. (1998) "Dinámica de una huella plana turbulenta alejada", Rev. Mat. Teor. Aplics. 5(1): 177-184.

[13] Townsend, A.A. (1956) The Structure of Turbulent Shear Flow. Cambridge University Press.

[14] Hanjalic, K.; Launder, B.E. (1972) "A Reynolds stress model of turbulence and its application to thin shear flows", J. Fluid Mech. 52: 609-638.

[15] Sabel'nikov, V. A. (1975) "Some specific feature of turbulent flows with zero excess momentum", Uch. Zap. TsAGI 6(4): 71-74 (in Russian). 
[16] Dmitrienko, Yu.M.; Kovalev, I.I.; Luchko, N.N.; Cherepanov, P.Ya. (1987) "Investigation of the plane turbulent wakes with zero excess momentum", Inzhenerno Fiz, Zh. 52(5): 743-751 (in Russian).

[17] Cimbala, J.M.; Park, W.J. (1990) "An experimental investigation of the turbulent structure in a two-dimensional momentumless wake", J. Fluid Mech. 213: 479-509.

[18] Chernykh, G.G.; Fedorova, N.N. (1994) "Numerical simulation of plane turbulent wakes", Math. Modelling 6(10): 14-24 (in Russian).

[19] Chernykh, G.G.; Demenkov, A.G. (1997) "On numerical simulation of jet flows of viscous incompressible fluids", Rus.J. Numer. Anal. Math. Modelling 12(2): 111125 .

[20] Cherepanov, P.Ya.; Babenko, V.A. (1998) "Experimental and numerical study of flat momentuumless wake", Int. J. Heat and Fluid Flow 19: 608-622.

[21] Kurbatsky, A.F. (1988) Modelling of Nonlocal Turbulent Transport of Momentum and Heat. Nauka, Novosibirs (in Russian).

[22] Samarskii, A.A.; Galaktionov, V.A.; Kurdyumov, S.P.; Mikhailov, A.P. (1995) Blowup in Quasiliniear Parabolic Equations. Walter de Gruyter, Berlin.

[23] Kraichnan, R. (1962) "The closure problem of turbulence theory", Proc. Symps. Appl. Math. 19: 199-225.

[24] Ilyushin, B.B. (1999) "Model of fourth-order cumulants for prediction of turbulent transport by large-scale vortex structure", J. Appl. Mechan. Tech. Phys. 40(5): 871876.

[25] Vázquez, J.L. (1985) "Hyperbolic aspects in the theory of the porous medium equation", Proceedings of the Workshop on Metastability and PDE's. Minnesota.

[26] Barenblatt, G. I. (1996) Scaling, selfsimilar and intermediate asymptotics. Cambridge Texts in Applied Mathematics, 14. 